\title{
Numerical Study of Invariant Sets of a Quasiperiodic Perturbation of a Symplectic Map
}

\author{
Stathis Tompaidis
}

\section{CONTENTS}

1. Introduction

2. Notation and Preliminaries

3. Existence and Computation of Periodic Orbits

4. Jacobi-Perron Approximation Schemes

5. Domains of Existence of Invariant Surfaces

6. Numerical Results

7. Critical Function

Acknowledgments

References
Partially supported by NSERC grant OGP-0121848 and European Community grant CHBGT-CT93-0244.

\begin{abstract}
We study the behavior of invariant sets of a volume-preserving map that is a quasiperiodic perturbation of a symplectic map, using approximation by periodic orbits. We present numerical results for analyticity domains of invariant surfaces, behavior after breakdown, and a critical function describing breakdown of invariant surfaces as a function of their rotation vectors. We discuss implications of our results to the existence of a renormalization group operator describing breakdown of invariant surfaces.
\end{abstract}

\section{INTRODUCTION}

The problem of existence and persistence of invariant sets of dynamical systems on which motion is, up to a smooth change of variables, quasiperiodic, has attracted considerable attention for at least a century [Poincaré 1892]. Such sets have many important, practical, applications as landmarks that organize the long-term behavior. For Hamiltonian systems that are close to integrable, the Kolmogorov-Arnol'd-Moser theorem guarantees that most such invariant sets persist for a small enough perturbation [Kolmogorov 1954; Arnol'd 1963; Moser 1962] (or see [Llave 1993] for a self-contained introduction to KAM theory and a proof). Results similar to the KAM theorem can also be shown in the case of quasiperiodic perturbations of symplectic maps.

Unfortunately, the available analytical estimates are very conservative compared to numerical indications, especially for high-dimensional systems. In this paper we investigate numerically the domains of existence of two-dimensional tori in a particular two-parameter family of volume-preserving 
maps, and the behavior that occurs at breakdown. The maps we study are quasiperiodic perturbations of a family of symplectic maps. Our numerical algorithms are based on analytical results described in [Falcolini and Llave 1992a] and [Tompaidis 1996] (the preceding article in this issue). The main idea is to use another landmark of longterm behavior, periodic orbits, to determine the existence and breakdown of tori.

For symplectic maps in two dimensions, it was originally observed in [Greene 1979] that existence of invariant circles has a strong influence on periodic orbits close to the circle. In [Tompaidis 1996] we prove that in symplectic maps of any dimension, as well as in quasiperiodic perturbations of them, existence of an invariant torus implies that the behavior of the map in a neighborhood of the torus is close to that of an integrable map. We will make use of this result as an indication of breakdown.

The system we will study is a three-dimensional model of a family of volume-preserving maps. Motion in one of the coordinates is rigid rotation with rotation number given by an appropriate diophantine number. The other two coordinates of the map are described by a perturbation of the standard map. Properties of the map and existence of tori have also been investigated in [Artuso et al. 1991]. Our rationale for studying this map (henceforth called the rotating standard map) is similar to that used in experimental physics, where one carefully prepares a sample in order to observe certain phenomena. In our case, the rotating standard map serves as a paradigm for phenomena that appear in higher-dimensional maps.

It is an important problem to describe the behavior at breakdown of invariant tori. In two dimensions, careful numerical experiments and analytical arguments suggest that the breakdown of invariant circles can be described by a fixed point (with a stable manifold of codimension one) of a renormalization-group operator [MacKay 1982].

We investigate whether such an approach generalizes to the case of the rotating standard map. To consider such a generalization we use a multidi- mensional algorithm (called the Jacobi-Perron algorithm) to approximate irrational points by rational ones. The algorithm reduces to the continuedfraction method for the case of one-dimensional invariant circles. Although it has not been widely used in the dynamical systems literature, it has important measure-theoretic and convergence properties, useful in introducing a renormalization-group operator. In [Kosygin 1991] such an operator was constructed, and it was shown that convergence to a (trivial) fixed point under repeated application of the operator implies existence of an invariant torus. We investigate whether breakdown can be understood in terms of a different fixed point.

In Section 2 we present the rotating standard map. In Section 3 we show existence of periodic orbits with any rotation vector and discuss efficient ways to compute them. In Section 4 we present the Jacobi-Perron algorithm and describe its connection to an extension of modular transformations. In Section 5 we discuss the computation of domains of existence for a particular invariant torus. In Section 6 we present numerical results, and discuss whether breakdown can be understood in terms of a fixed point of a renormalization-group operator in certain regions of parameter space. In Section 7 we generalize the notion of critical function.

\section{NOTATION AND PRELIMINARIES}

We will study a family of three-dimensional volumepreserving maps $F_{\varepsilon, k}: \mathbb{R} \times \mathbb{T}^{2} \rightarrow \mathbb{R} \times \mathbb{T}^{2}$, with $\varepsilon, k$ complex. The $F_{\varepsilon, k}$ are called rotating standard maps, and are defined as follows. For fixed $\varepsilon, k$, set

$A^{\prime}=A^{\prime}(A, \theta, \varphi)=A-\frac{1}{2 \pi}\left(k+\frac{\varepsilon}{2 \pi} \cos 2 \pi \varphi\right) \sin 2 \pi \theta$.

Then

$$
F_{\varepsilon, k}\left(\begin{array}{c}
A \\
\theta \\
\varphi
\end{array}\right)=\left(\begin{array}{c}
A^{\prime} \\
\theta+A^{\prime} \bmod 1 \\
\varphi+\omega_{2} \bmod 1
\end{array}\right) .
$$

The value of $\omega_{2}$ determines whether the standard map is perturbed periodically (for $\omega_{2}$ rational) or quasiperiodically (for $\omega_{2}$ irrational). 
We say that $\boldsymbol{x}$ is a periodic orbit of type $(P / N)$, for $P \in \mathbb{Z}^{2}$ and $N$ a positive integer, if $F_{\varepsilon, k}^{N}(\boldsymbol{x})=\boldsymbol{x}$ and $\tilde{F}_{\varepsilon, k}^{N}(\tilde{\boldsymbol{x}})=\tilde{\boldsymbol{x}}+(P, 0)$, where $\tilde{F}_{\varepsilon, k}, \tilde{\boldsymbol{x}}$ are (fixed) lifts of $F_{\varepsilon, k}, \boldsymbol{x}$ to the universal cover of $\mathbb{R} \times \mathbb{T}^{2}$. We will call $N$ the period of the orbit. For $d$-vectors we will use the norm $\|\boldsymbol{v}\|_{d}=\sum_{i=1}^{d}\left|v_{i}\right|$.

We define the rotation vector of an orbit of $\tilde{F}_{\varepsilon, k}$ as the two-dimensional vector

$$
\omega=\lim _{i \rightarrow \infty} \frac{\pi_{1}\left(\tilde{F}_{\varepsilon, k}^{i}(A, \theta, \varphi)\right)-(\theta, \varphi)}{i}
$$

if the limit exists, where $\pi_{1}$ the projection on the angle coordinates: $\pi_{1}(A, \theta, \varphi)=(\theta, \varphi)$. For a periodic orbit of type $(P / N)$ the rotation vector is $\omega=P / N$.

We are interested in the behavior of invariant sets with diophantine rotation vector. A diophantine rotation vector of type $(K, \tau)$ is a two-dimensional vector $\omega$ such that

$$
|P \cdot \omega| \geq \frac{K}{\|P\|_{2}^{\tau}} \quad \text { for } P \in \mathbb{Z}^{2}, P \neq 0, K>0 .
$$

It is well known [Arnol'd 1988] that, for some $K_{0}$ and fixed $K>K_{0}, \tau>1$ the set of vectors of type $(K, \tau)$ has positive Lebesgue measure in the unit square. In [Tompaidis 1996] we show that existence of invariant sets on which motion is conjugate to rigid rotation with diophantine rotation vector has certain implications for the properties of periodic orbits in the neighborhood of the invariant set. As in that paper we define the residue of a periodic orbit with period $N$

$$
R(\boldsymbol{x})=\frac{1}{6}\left(3-\operatorname{Tr}\left(D f^{N}(\boldsymbol{x})\right)\right) .
$$

Considering the lift of map (2.1) to the universal cover of $\mathbb{R} \times \mathbb{T}^{2}$ we write (2.1) in Lagrangian formulation as

$\theta_{n+1}-2 \theta_{n}+\theta_{n-1}=-\frac{1}{2 \pi}\left(k+\frac{\varepsilon}{2 \pi} \cos 2 \pi \varphi_{n}\right) \sin 2 \pi \theta_{n}$, $\varphi_{n+1}-2 \varphi_{n}+\varphi_{n-1}=0$,

where we set $\left(A_{n+1}, \theta_{n+1}, \varphi_{n+1}\right)=F_{\varepsilon, k}\left(A_{n}, \theta_{n}, \varphi_{n}\right)=$ $F_{\varepsilon, k}^{2}\left(A_{n-1}, \theta_{n-1}, \varphi_{n-1}\right)$. We will use these equations to numerically compute periodic orbits for $F_{\varepsilon, k}$.

\section{EXISTENCE AND COMPUTATION OF PERIODIC ORBITS}

John Mather [1991] extended several of the properties of twist maps of the annulus to finite compositions of twist maps. Consider a periodic perturbation of the standard map and the twist maps of the annulus

$$
f_{n}\left(\begin{array}{c}
p \\
q
\end{array}\right)=\left(\begin{array}{c}
p-s_{\varepsilon, k}\left(\varphi_{0}, \omega_{2}, n\right) \sin 2 \pi q \\
q+p-s_{\varepsilon, k}\left(\varphi_{0}, \omega_{2}, n\right) \sin 2 \pi q
\end{array}\right),
$$

where

$$
s_{\varepsilon, k}\left(\varphi_{0}, \omega_{2}, n\right)=\frac{1}{2 \pi}\left(k+\frac{\varepsilon}{2 \pi} \cos 2 \pi\left(\varphi_{0}+n \omega_{2}\right)\right) .
$$

The corresponding generating function is

$$
h_{n}\left(q, q^{\prime}\right)=\frac{1}{2}\left(q-q^{\prime}\right)^{2}-\frac{1}{\pi} s_{\varepsilon, k}\left(\varphi_{0}, \omega_{2}, n\right) \cos 2 \pi q .
$$

The finite composition

$$
f=f_{1} \circ \cdots \circ f_{N}
$$

is the rotating standard map with $B_{n} \equiv \omega_{2}=M / N$ and with generating function

$$
h=h_{1} * \cdots * h_{N},
$$

where

$$
h_{1} * h_{2}\left(x_{1}, x_{2}\right)=\min _{\xi}\left(h_{1}(x, \xi)+h_{2}\left(x^{\prime}, \xi\right)\right) .
$$

The operation $*$ was named conjunction in [Mather 1991] and used to show that many of the results for twist maps are preserved. In particular, for the rotating standard map as in (3.1) (a finite composition of twist maps), there exist configurations $\boldsymbol{x}$ for any $\omega \in \mathbb{R}$ such that $\boldsymbol{x}$ is an orbit of $f$ with rotation number $\omega$ [Mather 1991, Proposition 2.4]. Moreover, if $\Gamma$ is a curve invariant under $f$, on which motion is conjugate to rigid rotation with an irrational rotation number, then $\Gamma$ consists of minimal configurations of $h$. (For a discussion about minimal configurations see [Mather 1991]. For a proof see [Mather 1991, Proposition 2.8]). These results guarantee the existence of minimal periodic orbits for our maps, with any period and any (rational) rotation vector. 
To compute such periodic orbits for the map (2.1) we use Newton's method in the space of finite length sequences. Given a periodic orbit with rotation vector $\left(P_{1} / N, P_{2} / N\right)$ we can eliminate the dependence on the $\varphi$ variable in (2.3) and transform the equations to

$$
\theta_{n+1}-2 \theta_{n}+\theta_{n-1}=-s_{\varepsilon, k}\left(\varphi_{0}, P_{2} / N, n\right) \sin 2 \pi \theta_{n} .
$$

Equations (3.4) can be seen as the Euler-Lagrange equations for a certain Lagrangian [Mather 1991; Kook and Meiss 1989]. We introduce (formally) the action $W$ for an orbit $\left\{\theta_{i}\right\}_{i=-\infty}^{\infty}$ by

$$
W\left(\ldots, y_{i}, \ldots\right)=\sum_{n=-\infty}^{\infty} g\left(y_{n}, y_{n+1}, n\right)
$$

where $y$ is the lift of $\theta$ and

$$
g(x, y, i)=\frac{1}{2}(x-y)^{2}-\frac{1}{2 \pi} s_{\varepsilon, k}\left(\varphi_{0}, \frac{P_{2}}{N}, i\right) \cos 2 \pi x .
$$

Even though $W$ is only formally defined, its gradient is well defined, and a sequence of points is an orbit of (2.1) if and only if it is a critical point of $W$ [Kook and Meiss 1989; Golé 1994]. In coordinates, this condition is

$$
\frac{\partial}{\partial y_{l}}\left(g\left(y_{l-1}, y_{l}, l-1\right)+g\left(y_{l}, y_{l+1}, l\right)\right)=0
$$

for all $l$.

A minimal configuration for the generating function (3.2) corresponds to a critical point for $W$, but not necessarily vice versa, since minimax configurations may exist for certain values of the parameters.

For a periodic orbit of type $\left(P_{1} / N, P_{2} / N\right)$ we have $y_{N+1}=y_{1}+P_{1} / N, y_{0}=y_{N}-P_{1} / N$. For this case we redefine the action $W$ as

$$
W_{N}\left(y_{1}, \ldots, y_{N}\right)=\sum_{n=1}^{N} g\left(y_{n}, y_{n+1}, n\right),
$$

which leads to the system

$$
\frac{\partial}{\partial y_{l}}\left(g\left(y_{l-1}, y_{l}, l-1\right)+g\left(y_{l}, y_{l+1}, l\right)\right)=0
$$

for $l=1, \ldots, N$. To solve this system, consider the operator $\mathcal{T}: \mathbb{R}^{N} \rightarrow \mathbb{R}^{N}$ given by

$$
\mathcal{T}[\boldsymbol{y}]=\frac{\partial}{\partial y_{l}} W_{N}, \quad \text { for } l=1, \ldots, N .
$$

Given an initial guess for the coordinates of the periodic orbit $\boldsymbol{y}=\left(y_{1}, \ldots, y_{N}\right)$, we can improve it by setting $\boldsymbol{y}^{\prime}=\boldsymbol{y}+\delta y$. Ignoring terms of order $\delta y \cdot \delta y$ we have

$$
D \mathcal{T}[\boldsymbol{y}] \delta y=-\mathcal{T}[\boldsymbol{y}],
$$

or

$$
\begin{aligned}
A \delta y & =\left(\begin{array}{ccccc}
2-a_{1} & -1 & \ldots & 0 & -1 \\
-1 & 2-a_{2} & \ldots & 0 & 0 \\
\vdots & \vdots & \ddots & \vdots & \vdots \\
0 & 0 & \ldots & 2-a_{N-1} & -1 \\
-1 & 0 & \ldots & -1 & 2-a_{N}
\end{array}\right)\left(\begin{array}{c}
\delta y_{1} \\
\delta y_{2} \\
\vdots \\
\delta y_{N-1} \\
\delta y_{N}
\end{array}\right) \\
& =-\mathfrak{T}[\boldsymbol{y}],
\end{aligned}
$$

where

$$
a_{i}=2 \pi s_{\varepsilon, k}\left(\varphi_{0}, \frac{P_{2}}{N}, i\right) \cos 2 \pi y_{i} .
$$

This method of finding periodic orbits is very similar to the one used in [Kook and Meiss 1989] for the case of high-dimensional symplectic maps. The only difference is that the coefficients $a_{i}$ in our case depend not only on the coordinates of the periodic orbit, but on the iteration number itself. Another possible method for numerically computing certain periodic orbits of maps with symmetries uses properties of periodic orbits with respect to symmetry lines of the map. We have not made use of symmetry lines in our numerical algorithm.

Our numerical implementation of the method for a particular $\left(P_{1} / N, P_{2} / N\right)$ periodic orbit used the following continuation algorithm:

- Choose a precision cutoff value $\delta$. (In our computations we chose $\delta=10^{-7}$.)

- Choose a family of paths passing through $k=$ $\varepsilon=0$.

- For a point along a path iterate Newton's algorithm, using as initial guess the periodic orbit computed at the previous point on the path. 
- Proceed to the next point when the error between the computed periodic orbit and its first iterate is less than $\delta$.

\section{Iterative Methods}

The problem of solving the linear system (3.5) is greatly simplified by the fact that the matrix $A$ is sparse, with only $3 N$ nonzero entries. To solve systems of linear equations involving large, sparse matrices, iterative methods have been developed (similar problems appear frequently in finite-element and finite-difference discretizations of partial differential equations). Iterative methods have the advantage that, for sparse matrices, only $O(N)$ computation steps and storage space are required.

Given the linear system $A u=b$ to be solved, an iterative method successively approximates the true solution $\bar{u}$ from an initial guess $u^{(0)}$. The iteration scheme is

$$
u^{(n+1)}=G u^{(n)}+k,
$$

where $G=I-Q^{-1} A$ and $k=Q^{-1} b$, for a suitable matrix $Q$. This matrix is chosen so as to be invertible in $O(N)$ steps (this is the case for diagonal, tridiagonal, and triangular matrices).

An iterative method can be further speeded up by the use of an acceleration procedure. Such procedures are based on properties of the matrix $A$. For example, Chebyshev acceleration uses information about the estimated range of the eigenvalues of $A$, whereas the conjugate gradient method minimizes a certain function of $A$. For a more complete description, with many examples, see [Young and Gregory 1988; Young and Hageman 1981]. Convergence of an iterative method is checked by monitoring the norm of the error.

Convergence of some iterative methods to the true solution has been rigorously demonstrated for the case where $A$ is a symmetric, positive definite matrix. Unfortunately we are not aware of any general convergence result for matrices that are not symmetric.

In our computations we have used the package ITPACK 2C [Kincaid et al. 1982], developed at the Center for Numerical Analysis of the University of Texas at Austin. We used the Jacobi iterative method with either Chebyshev acceleration or conjugate gradient acceleration; for $Q$ we used the diagonal part of $A$. We verified that the time to converge to a solution, within a specified precision, increased linearly with $N$ for a periodic orbit of type $\left(P_{1} / N, P_{2} / N\right)$.

\section{Direct Methods}

In [Kook and Meiss 1989] a direct algorithm was proposed to solve an equation similar to (3.5) in the case of high-dimensional symplectic maps. The algorithm had the advantages of an iterative method in that it required only $O(N)$ steps and $O(N)$ storage space. We implemented a similar method, taking advantage of the structure of the matrix $A$.

The method is based on the fact that $A$ is very close to a tridiagonal matrix, namely it is cyclic tridiagonal (tridiagonal with two additional entries at the corners). We first solve the tridiagonal linear system in $O(N)$ steps using the Thomas algorithm (see [Young and Gregory 1988, vol. 2, p. 587]). The algorithm takes $O(N)$ computational steps to perform Gaussian elimination, due to the special form of a tridiagonal matrix. After finding the solution to the tridiagonal problem we can add corrections, due to the terms at the two corners, either using the Sherman-Morrison method or the Woodbury method [Press et al. 1992, p. 73-77].

The algorithm can be easily extended to higherdimensional cases. Our implementation is designed to be flexible, so that periodic orbits in different maps (either volume-preserving or symplectic) and even higher-dimensional systems can be computed by simply changing some map-dependent definitions in a file.

\section{JACOBI-PERRON APPROXIMATION SCHEMES}

Approximation of irrational numbers by rationals has been important in the study of breakdown of invariant curves in twist maps of the annulus since the work of Greene [1979]. Greene conjectured that 
the behavior of periodic orbits with rotation numbers that are continued-fraction convergents of a diophantine irrational number determines the existence of an invariant curve with rotation number equal to the irrational. MacKay [1982] constructed a renormalization-group operator on spaces of analytic maps, which changes the rotation number of an invariant curve by eliminating the first continued fraction coefficient. For irrationals with periodic continued fraction expansions - and in particular for the golden mean $\gamma=\frac{1}{2}(\sqrt{5}-1)$-he found evidence, based on careful numerical work and analytical arguments, that the breakdown of invariant curves is described by a fixed point of the renormalization operator with a stable manifold of codimension one.

\section{Description of the Algorithm}

The Jacobi-Perron algorithm is one of many generalizations of the continued fraction algorithm in higher dimensions. Detailed description of the algorithm and proofs of the results we present here can be found in [Bernstein 1971; Schweiger 1973; Kosygin 1991; Lagarias 1993].

Given a point $\left(\omega_{1}, \omega_{2}\right) \in(0,1) \times(0,1)$, the algorithm recursively defines convergents $P_{n} / N_{n}$, with $P_{n}=\left(P_{1_{n}}, P_{2_{n}}\right) \in \mathbb{N}^{2}$ and $N_{n} \in \mathbb{N}$. Their definition is

$$
\begin{aligned}
& P_{n+1}=k_{n+1} P_{n}+l_{n+1} P_{n-1}+P_{n-2}, \\
& N_{n+1}=k_{n+1} N_{n}+l_{n+1} N_{n-1}+N_{n-2},
\end{aligned}
$$

where the integer coefficients $k_{n+1}, l_{n+1}$ are determined by the Jacobi-Perron map

$$
\begin{aligned}
\left(\omega_{1}^{n+1}, \omega_{2}^{n+1}\right) & =\left(\frac{1}{\omega_{2}^{n}} \bmod 1, \frac{\omega_{1}^{n}}{\omega_{2}^{n}} \bmod 1\right), \\
\left(k_{n+1}, l_{n+1}\right) & =\left(\left\lfloor\frac{1}{\omega_{2}^{n}}\right\rfloor,\left\lfloor\frac{\omega_{1}^{n}}{\omega_{2}^{n}}\right\rfloor\right),
\end{aligned}
$$

with initial values $\left(\omega_{1}^{0}, \omega_{2}^{0}\right)=\left(\omega_{1}, \omega_{2}\right), P_{0}=(0,0)$, $P_{-1}=(1,0), P_{-2}=(0,1), N_{0}=1, N_{-1}=N_{-2}=0$.

The Jacobi-Perron algorithm is a linear simplexsplitting algorithm [Lagarias 1993]. For the case of points in the unit square a consequence is that if three successive Jacobi-Perron approximants define a triangle, all approximants of higher order (and the point being approximated) will lie inside that triangle. This property is shared with another commonly used algorithm, the Farey-tree approximation scheme [Kim and Ostlund 1986]. Lagarias studied the rate of convergence of the Jacobi-Perron algorithm for a set of points of Lebesgue measure one in the unit square. Consider a point in the unit square $w$ and a close-by point with rational coordinates $r=\left(p_{1} / q, p_{2} / q\right)$. The Roth exponent of $r$ is defined as

$$
\eta(r, w)=-\frac{\log \|w-r\|_{2}}{\log q} .
$$

Let $r_{i}$ be the $i$-th Jacobi-Perron approximant to a point $w$. The best approximation exponent for $w$ using the Jacobi-Perron scheme is defined as

$$
\eta_{b} \equiv \limsup _{i \rightarrow \infty}\left\{\eta\left(r_{i}(w)\right)\right\}
$$

and the uniform approximation exponent as

$$
\eta_{u} \equiv \liminf _{i \rightarrow \infty}\left\{\min \left(\eta\left(r_{i}(w)\right), \eta\left(r_{i+1}(w)\right), \eta\left(r_{i+2}\right)\right)\right\} .
$$

The exponent $\eta_{b}$ gives the rate of convergence for the best possible approximant towards a point, while the exponent $\eta_{u}$ is an (asymptotic) estimate for the rate at which all the vertices of the triangle enclosing $w$ approach $w$.

Lagarias showed, using methods from ergodic theory (see [Lagarias 1993], also [Kosygin 1991]) that for the Jacobi-Perron algorithm $\eta_{b}$ and $\eta_{u}$ are constant in a set of measure one in the unit square. He conjectured that the constant values are in fact equal. In [Baldwin 1992] (see also [Kosygin 1991]) numerical methods were used to estimate $\eta_{b}=1.374 \pm 0.002$, an estimate that, coupled with Lagarias' conjecture, suggests that the triangles formed from successive Jacobi-Perron approximants become, in the limit, needle-shaped.

The Jacobi-Perron algorithm has other properties of interest, apart from its measure-theoretic ones. For all points in the unit square, we have $k_{n} \geq 1$ and $k_{n} \geq l_{n} \geq 0$. Also, the triangle 
formed by three successive Jacobi-Perron approximants contains no rational point with denominator smaller than the largest denominator of the vertices.

In analogy with periodic continued fractions of period 1, we introduce golden means of the JacobiPerron algorithm $\omega$ for which $k_{n}=k, l_{n}=l$. The polynomial $P_{\omega}(t)=t^{3}-k t^{2}-l t-1$ is called the characteristic polynomial of $\omega$. Golden means have the following properties:

- $k<\tau<k+1,0<\left|\tau_{1}\right|$, and $\left|\tau_{2}\right|<1$, where $\tau$ is the root of $P_{\omega}$ of maximal absolute value and $\tau_{1}, \tau_{2}$ are the remaining roots (so that $\left|\tau_{1}\right|,\left|\tau_{2}\right|<1$ ).

- $\left(\omega_{1}, \omega_{2}\right)=(\tau-k, 1 / \tau)$.

- $\left\|q_{n} \omega-\left(p_{1_{n}}, p_{2_{n}}\right)\right\|_{2} \leq C(\omega) \max \left(\left|\tau_{1}\right|,\left|\tau_{2}\right|\right)^{n}$.

Kosygin [1991] has constructed a renormalizationgroup operator on the space of symplectic maps of $\mathbb{R}^{2} \times \mathbb{T}^{2}$. The action of the operator on a map that has an invariant surface with rotation vector

$$
\left(\omega_{1}, \omega_{2}\right)=\left(\left(k_{1}, l_{1}\right),\left(k_{2}, l_{2}\right), \ldots\right)
$$

produces a new map with an invariant surface with rotation vector $\left(\omega_{1}^{\prime}, \omega_{2}^{\prime}\right)=\left(\left(k_{2}, l_{2}\right), \ldots\right)$. For golden means of the Jacobi-Perron algorithm he showed that if the original map, under repeated action of the renormalization-group operation, converges to a (trivial) map (which is a fixed point of the renormalization-group operator), then the original map admits an invariant surface, on which motion is conjugate to rigid rotation with rotation vector $\left(\omega_{1}, \omega_{2}\right)$. Moreover, the trivial fixed point is attractive, that is, maps in its neighborhood admit an invariant surface. (This result can also be viewed as a renormalization-group proof of the KAM theorem for invariant surfaces with rotation vectors golden means of the Jacobi-Perron algorithm.)

\section{Connection Between the Jacobi-Perron Algorithm and} $\mathrm{PSL}_{3}(\mathbb{Z})$

The Jacobi-Perron algorithm has a natural connection with $3 \times 3$ matrix transformations. We introduce an extension of modular transformations of one complex variable to two complex variables:

$$
\left(z_{1}, z_{2}\right) \mapsto\left(\frac{a z_{1}+b z_{2}+c}{g z_{1}+h z_{2}+i}, \frac{d z_{1}+e z_{2}+f}{g z_{1}+h z_{2}+i}\right) .
$$

Successive transformations obey the rules of matrix multiplication of $3 \times 3$ matrices

$$
M=\left(\begin{array}{lll}
a & b & c \\
d & e & f \\
g & h & i
\end{array}\right)
$$

Because of invariance of the transformation under scaling, we can assume $|\operatorname{det} M|=1$, where $M$ and $-M$ are identified. Thus the group of transformations defined by (4.3) is isomorphic to the projective group $\mathrm{PSL}_{3}(\mathbb{Z})$.

$\mathrm{PSL}_{3}(\mathbb{Z})$ is generated by the elementary matrices $T_{i j}=I+e_{i j}$, for distinct $i, j=1,2,3$, where $e_{i j}$ is the $3 \times 3$ matrix whose only nonzero entry is the element $i, j$, which is equal to 1 . On the other hand, the Jacobi-Perron operator can be viewed as a subgroup of $\mathrm{PSL}_{3}(\mathbb{Z})$ generated by the matrices

$$
T_{1}=\left(\begin{array}{lll}
1 & 0 & 1 \\
0 & 1 & 0 \\
0 & 0 & 1
\end{array}\right), T_{2}=\left(\begin{array}{lll}
1 & 0 & 0 \\
0 & 1 & 1 \\
0 & 0 & 1
\end{array}\right), U=\left(\begin{array}{lll}
0 & 1 & 0 \\
0 & 0 & 1 \\
1 & 0 & 0
\end{array}\right) .
$$

$T_{1}$ represents translation in the first coordinate, $T_{2}$ translation in the second coordinate, and $U$ generalized inversion.

Given a point $\left(\omega_{1}, \omega_{2}\right)$ in the unit square, the Jacobi-Perron operator can be written as

$$
\begin{aligned}
\left(\omega_{1}^{(0)}, \omega_{2}^{(0)}\right) & =\left(\omega_{1}, \omega_{2}\right), \\
\left(\omega_{1}^{(n+1)}, \omega_{2}^{(n+1)}\right) & =T_{1}^{-k_{n}} T_{2}^{-l_{n}} U\left(\omega_{1}^{(n)}, \omega_{2}^{(n)}\right) \text { for } n \geq 1,
\end{aligned}
$$

where $k_{n}, l_{n}$ are the unique nonnegative integers such that $\left(\omega_{1}^{(n+1)}, \omega_{2}^{(n+1)}\right)$ is inside the unit square. The coefficients $k_{n}, l_{n}$ form the Jacobi-Perron expansion of $\left(\omega_{1}, \omega_{2}\right)$.

We can use the Jacobi-Perron operator to move between points with the same tail coefficients, since $\omega^{(0)}=\left(\left(k_{0}, l_{0}\right),\left(k_{1}, l_{1}\right), \ldots\right)$ implies

$$
\omega^{(n)}=\left(\left(k_{n}, l_{n}\right),\left(k_{n+1}, l_{n+1}\right), \ldots\right) .
$$

The difference between this case and the lowerdimensional case studied in [Buric et al. 1990] is 
that the Jacobi-Perron transformation corresponds to a subgroup of $\mathrm{PSL}_{3}(\mathbb{Z})$, whereas the continued fraction algorithm corresponds to $\mathrm{PSL}_{2}(\mathbb{Z})$.

\section{DOMAINS OF EXISTENCE OF INVARIANT SURFACES}

Existence of invariant surfaces on which motion is, up to a change of variables, rigid rotation, plays a significant role in determining long-term dynamics in many physical applications. In the case of the rotating standard map the existence of twodimensional invariant tori presents a complete barrier to phase-space diffusion. Perry and Wiggins [1994] showed that invariant tori in high-dimensional Hamiltonian systems also guarantee longterm stability for orbits in their neighborhood.

Several methods, both analytical and numerical, have been used in two-dimensional systems to determine the domain of existence of invariant curves [Celletti and Chierchia 1988; Rana 1987; Llave and Rana 1991; Berretti and Chierchia 1990; Falcolini and Llave 1992b]. Unfortunately, estimates based on analytical methods are very conservative in the case of higher-dimensional systems. Among the numerical methods for two-dimensional systems, the one widely believed to be the most accurate is based on a conjecture of Greene concerning the behavior of periodic orbits approaching the invariant curve (see [Greene 1979], and also [Falcolini and Llave 1992a; MacKay 1992] for a rigorous, partial justification).

Results in [Falcolini and Llave 1992a; Tompaidis 1996] provide justification for a similar criterion in higher-dimensional models. Following the latter paper (which can be found in this issue), we will consider volume-preserving maps that are quasiperiodic skew products of symplectic maps over $\mathbb{T}^{e}$, that is, maps of the form

$$
f(\theta, \varphi, A)=\left(f_{1}(\theta, \varphi, A), \varphi+\omega_{2}, f_{2}(\theta, \varphi, A)\right),
$$

for $f_{1}: \mathbb{T}^{d+e} \times \mathbb{R}^{d} \rightarrow \mathbb{T}^{d}, f_{2}: \mathbb{T}^{d+e} \times \mathbb{R}^{d} \rightarrow \mathbb{R}^{d}$, $\theta \in \mathbb{T}^{d}, \varphi \in \mathbb{T}^{e}$, and $\omega_{2} \in \mathbb{T}^{e}$ an irrational vector.
We introduce the extension $f^{*}: \mathbb{T}^{d+e} \times \mathbb{R}^{d+e} \rightarrow$ $\mathbb{T}^{d+e} \times \mathbb{R}^{d+e}$ by

$$
\begin{array}{r}
f^{*}\left(\theta, \varphi, A_{1}, A_{2}\right) \\
=\left(f_{1}\left(\theta, \varphi, A_{1}\right), \varphi+A_{2}, f_{2}\left(\theta, \varphi, A_{1}\right), A_{2}\right),
\end{array}
$$

which at $A_{2}=\omega_{2}$ reduces to $f$. If $f$ admits an invariant surface $\Gamma$ then $f^{*}$ admits an invariant surface $\Gamma^{*}$ at $A_{2}=\omega_{2}$. Moreover we introduce the restriction $f_{\omega}^{*}: \mathbb{T}^{d+e} \times \mathbb{R}^{d} \rightarrow \mathbb{T}^{d+e} \times \mathbb{R}^{d}$, where $\omega \in \mathbb{T}^{e}$, by setting

$$
f_{\omega}^{*}(\theta, \varphi, A)=f^{*}(\theta, \varphi, A, \omega) .
$$

If $f^{*}$ admits a periodic orbit $x$ of type $\left(\left(P_{1}, P_{2}\right) / N\right)$ then $f_{P_{2} / N}^{*}$ admits a periodic orbit $\bar{x}$ of the same type.

Theorem 5.1 [Tompaidis 1996, Theorem 2.4]. Let $f: \mathbb{T}^{d+e} \times \mathbb{R}^{d} \rightarrow \mathbb{T}^{d+e} \times \mathbb{R}^{d}$ be a quasiperiodic skewproduct of a $2 d$-dimensional nonsingular symplectic map over $\mathbb{T}^{e}$ such that $\left.f\right|_{\mathbb{T}^{e}}$ is rigid rotation with a diophantine rotation vector. Assume that $f$ is of class $C^{r}$, where $r>1$, and that it admits a $C^{r}$ invariant surface $\Gamma$, homotopic to $\mathbb{T}^{d+e} \times\{0\}$, on which the motion is $C^{r}$ conjugate to rigid rotation with rotation vector $\omega$ of type $(K, \tau)$. Moreover, assume that in the extension $f^{*}$ of $f$ there is a neighborhood of $\Gamma^{*}$ where there are periodic orbits $x_{(P / N)}\left(P \equiv\left(P_{1}, P_{2}\right) \in \mathbb{Z}^{d+e}\right)$ of type $(P / N)$ for $\|N \omega-P\|_{d+e}$ small enough. Then, for any nonnegative integer $k<(r-1) / \tau$, we can find $D_{k}>0$ such that $2 d$ of the eigenvalues $\lambda_{1}, \ldots, \lambda_{2 d}$ of the derivative $D\left(\left(f_{P_{2} / N}^{*}\right)^{N}\right)\left(\bar{x}_{(P / N)}\right)$ satisfy

$$
\left|\lambda_{i}-1\right| \leq D_{k}\|N \omega-P\|_{d+e}^{k / 2} N \quad \text { for } i=1, \ldots, 2 d,
$$

the remaining e eigenvalues being identically 1 .

If $f, \Gamma$, and the conjugacy to rigid rotation are analytic, we can find $\tilde{C}_{1}, \tilde{C}_{2}>0$ such that

$$
\left|\lambda_{i}-1\right| \leq \tilde{D}_{1} N \exp \left(-\tilde{D}_{2}\|N \omega-P\|_{d+e}^{-1 /(2(1+\tau))}\right) .
$$

Remark. For the rotating standard map the eigenvalues of the derivative are completely determined by the trace (since one eigenvalue is identically 1 
and the map is volume-preserving). Therefore, instead of monitoring the eigenvalues, we will study the behavior of the residue

$$
R(\boldsymbol{x})=\frac{1}{6}\left(3-\operatorname{Tr}\left(D f^{N}(\boldsymbol{x})\right)\right)
$$

along a periodic orbit $\boldsymbol{x}$ with period $N$.

Remark. In the case of hyperbolic invariant sets that are semi-conjugate to rigid rotation, it was shown in [Falcolini and Llave 1992a] that the derivative along periodic orbits approaching the invariant set has eigenvalues that increase exponentially with the period of the orbit. Although the invariant sets of the rotating standard map are not hyperbolic, due to rigid rotation in the second angle coordinate, we have observed that in the absence of an invariant surface the eigenvalues of periodic orbits approaching a limit set are exponentially increasing.

Theorem 5.1 (which also holds for complex maps and complex invariant sets) suggests the following algorithm:

- Fix a value $\alpha>0$, to be used as the cutoff criterion for determining breakdown.

- Choose a family of paths in the parameter space.

- Compute periodic orbits with rotation vectors close to the rotation vector of an invariant set of interest, along the paths, and determine the point along the path when the residue of the periodic orbit satisfies $|R(x)|>\alpha$.

Remark. The value of $\alpha$ plays only a minor role if the period is large. The reason is that if an invariant curve exists, the eigenvalues of the derivative of the map approach 1 exponentially as the period increases, whereas if the invariant surface has "disintegrated" then at least one eigenvalue is expected to be exponentially large. The reasoning is valid as long as the breakdown of an invariant surface is well-defined.

Remark. The algorithm is based on continuation of periodic orbits using Newton's method in a space of finite sequences, as presented in Section 3. Using numerically computed condition numbers we can determine the validity of the continuation scheme. As long as no eigenvalue of the derivative of the map along the periodic orbit is 1 , the implicit function theorem guarantees the success of the continuation method for small enough steps along the path. Notice that one eigenvalue in the case of the rotating standard map is identically 1 , due to rigid rotation in the second angle coordinate, but does not influence Newton's method, as described by $(3.5)$.

\section{NUMERICAL RESULTS}

In Figures 1-3 we follow periodic orbits along paths in the parameter plane. We start the paths at $\varepsilon=k=0$, where we know the coordinates of the periodic orbits. The initial value of $\varphi_{0}$ is taken to be $\frac{1}{2}$. In Figure 1 we investigate the domain of existence of the invariant curve with rotation vector $\omega$ with Jacobi-Perron expansion $(1,1)^{\infty}$ for real values of $k, \varepsilon$. We observe that the boundaries for successive approximants cross each other for several sets of parameter values (a similar phenomenon was observed in [Falcolini and Llave 1992b] for standard-like maps in the complex domain). The phenomenon is more pronounced for large values of $k$ (Figure 2). In [Artuso et al. 1991] something similar was observed for a different invariant surface (with rotation vector the spiral mean) using a different approximation scheme (an extension of the Farey-tree expansion). We observed, however, that for certain paths in the parameter plane the boundaries for successive approximants are well-ordered and their successive positions follow a power law. Such paths are the $\varepsilon=0$ path (corresponding to the well-understood case of the standard map, albeit for the two-dimensional Jacobi-Perron and not the continued fraction approximation scheme) and the $k=0$ path; see Figure 4.

Figure 3 shows the domain of analyticity of the same invariant surface for a small, fixed value of $k$ and for complex values of $\varepsilon$. The absence of crossings and the scaling observed in the figure suggests that behavior at breakdown could be understood 


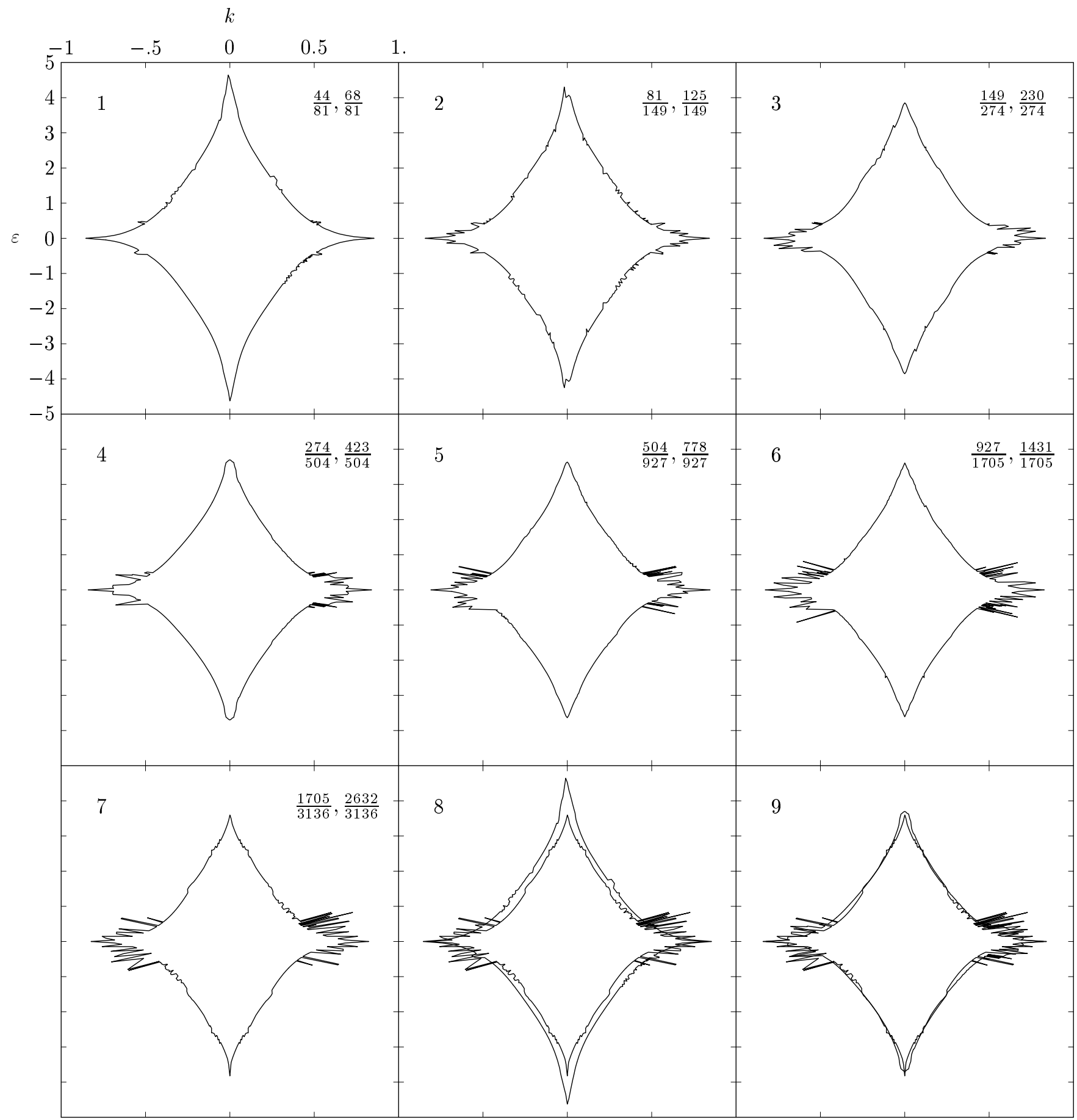

FIGURE 1. Domain of existence for the invariant surface with golden rotation vector $(1,1)^{\infty}$, for real values of the parameters. Plots 1-7 represent the boundary where the residue of periodic orbits is 1 , for the given values of the rotation vector, which were chosen as Jacobi-Perron convergents to $(1,1)^{\infty}$. Plot 8 is a superimposition of 1 and 7 , and 9 is a superimposition of 4 and 7 . The ranges of $k$ and $\varepsilon$ are the same in all plots. 


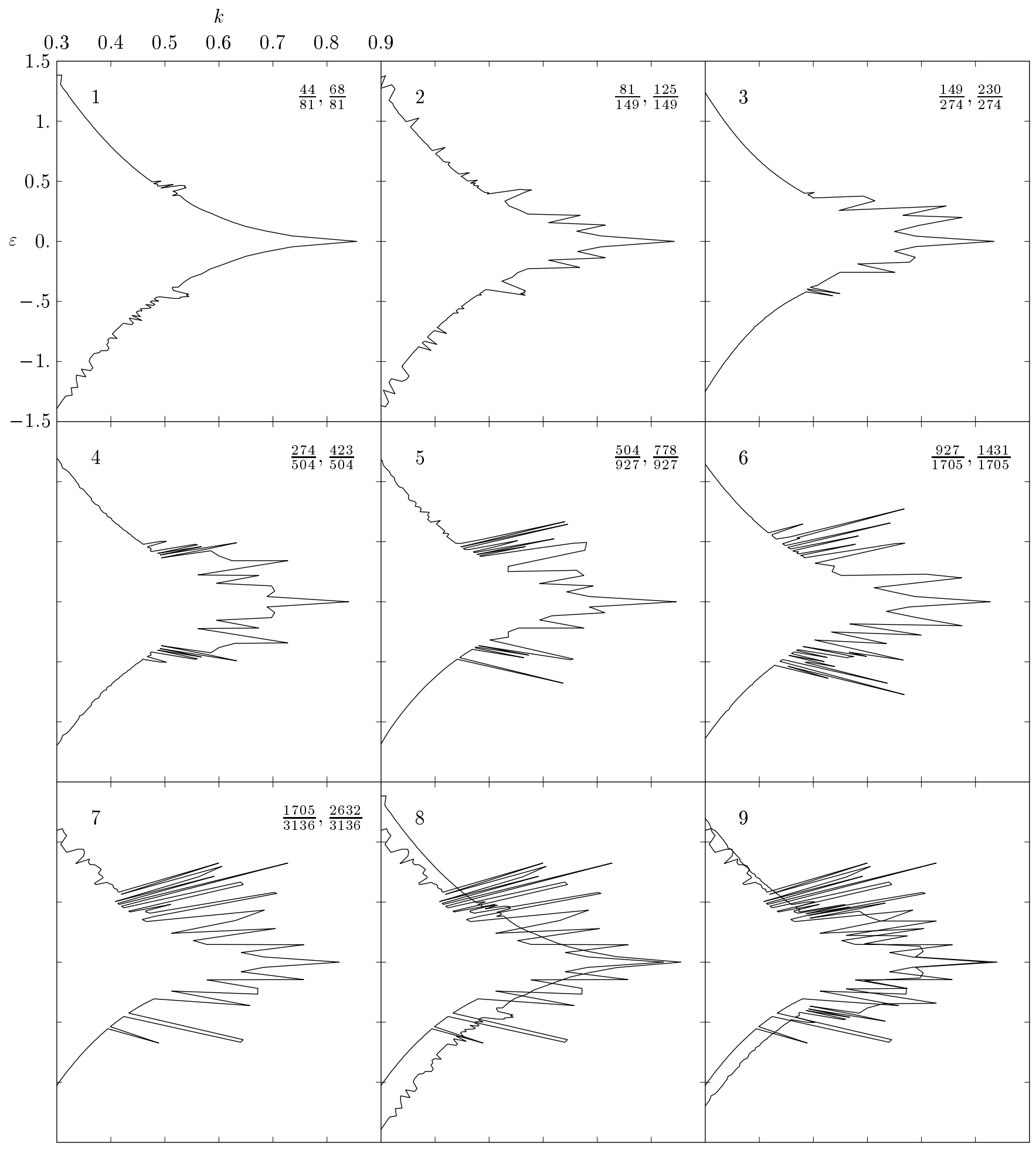

FIGURE 2. Detail of Figure 1. 


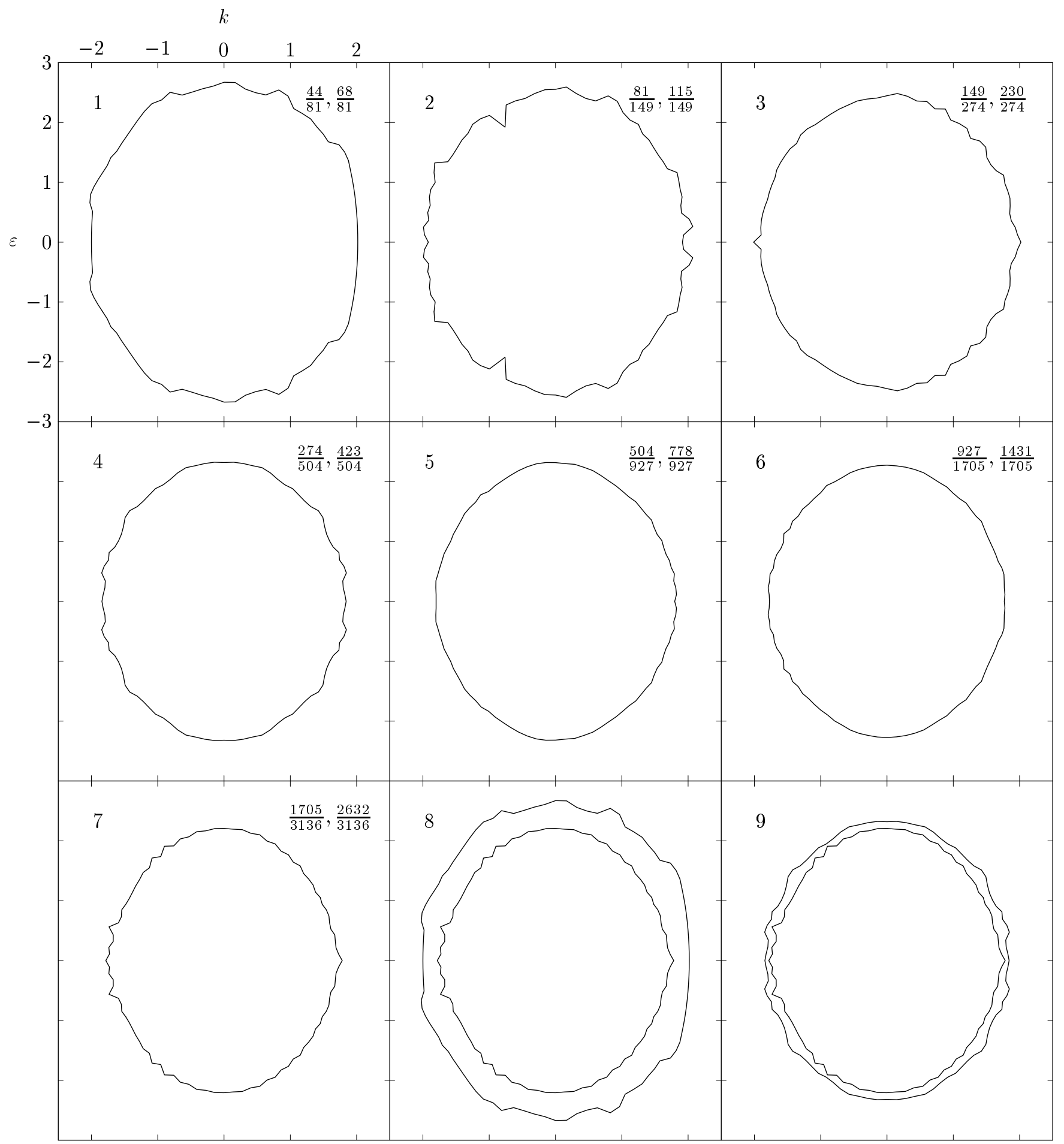

FIGURE 3. Domain of existence for the invariant surface with golden rotation vector $(1,1)^{\infty}$ for $k=0.2$ fixed and $\varepsilon$ complex. Plot 8 is a superimposition of 1 and 7 , and 9 is a superimposition of 4 and 7 . 


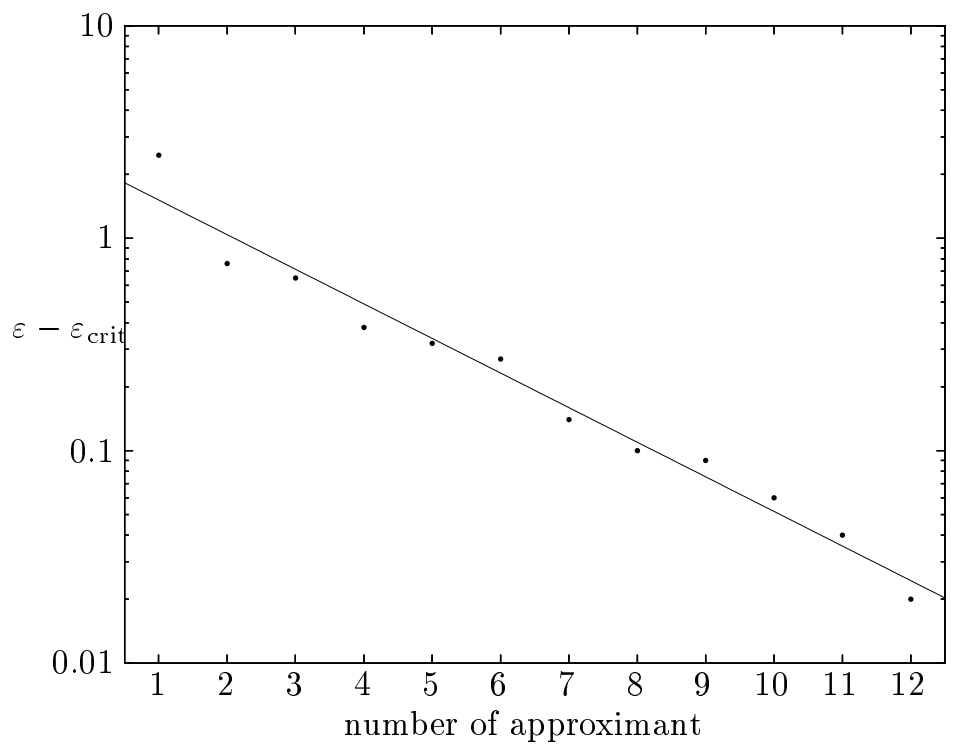

FIGURE 4. Scaling of the points where the residue becomes 1 for $k=0$, for successive Jacobi-Perron approximants to $(1,1)^{\infty}$. The first approximant is $\left(\frac{7}{13}, \frac{11}{13}\right)$ and the last is $\left(\frac{5768}{10609}, \frac{8904}{10609}\right)$. The value of $\varepsilon_{\text {crit }}$ and the approximating straight line are determined from a least squares Levenberg-Marquardt scheme [Press et al. 1992]; the line has equation $y=2.2 e^{-0.375 x}$.

in terms of a fixed point of a renormalization operator in a space of maps with complex variables.

For invariant surfaces that break down to invariant sets semi-conjugate to rotation, we can study the transition by monitoring the behavior of closeby periodic orbits. It is natural to ask whether an analog of Aubry-Mather theory applies to higherdimensional systems and what are the invariant sets that an invariant surface breaks down to. The continuation algorithm allows to compute periodic orbits of high period close to breakdown. Based on Figure 1 we identified breakdown of the invariant surface with rotation vector $(1,1)^{\infty}$ at $\varepsilon=1.75$, $k=0.2$ and at $\varepsilon=3.55, k=0$. We remark that although crossings between boundaries where different periodic orbits had residue 1 were common, the residue of each periodic orbit along a path appeared to behave regularly.

The transition occurring at breakdown was investigated in Figure 5. We observe that the invariant set develops gaps, analogous to the ones that appear as an invariant curve breaks down to an Aubry-Mather set in two-dimensional systems. Such gaps have been used to show rigorously, by means of a computer-assisted proof, that breakdown has occurred (see [Muldoon 1989] for the case of a four-dimensional symplectic map). Notice that the largest gap occurs at $\theta=\varphi=0$ where the potential attains its maximum.

Remark. For the case of the rotating standard map we have shown in Section 3 that periodic orbits for all rational rotation vectors exist. However we have not been able to rigorously determine whether they converge to an invariant set as their rotation vector tends to a diophantine rotation vector.

Figure 6 shows the behavior of the residue along a path in parameter space. In Figures 7 and 8 we investigate the behavior, along two paths in parameter space, of the stability of periodic orbits, quantified by the stability exponent $\lambda$. This exponent is defined, for an orbit $x$ of period $N$, as

$$
\lambda=\frac{1}{N} \log \left|D f^{N}(x)\right|,
$$

where $\left|D f^{N}(x)\right|$ means the biggest eigenvalue of the matrix. The stability exponent tends, in the 


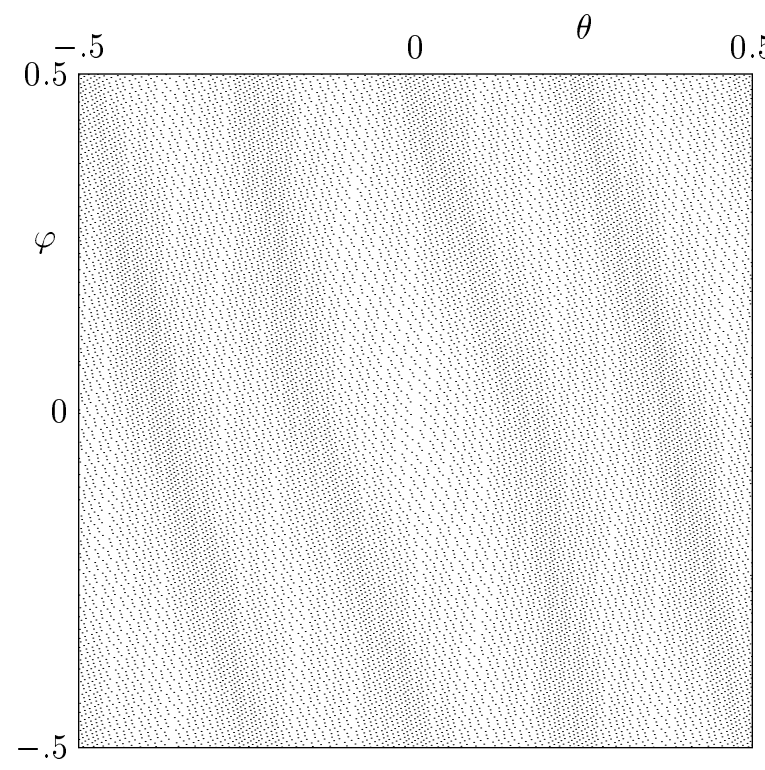

0.5

$k=0.2$

$\varepsilon=1.7$

$R=-0.831$

$$
\begin{array}{r}
k=0.2 \\
\varepsilon=1.8 \\
R=-7.01 \times 10^{12}
\end{array}
$$

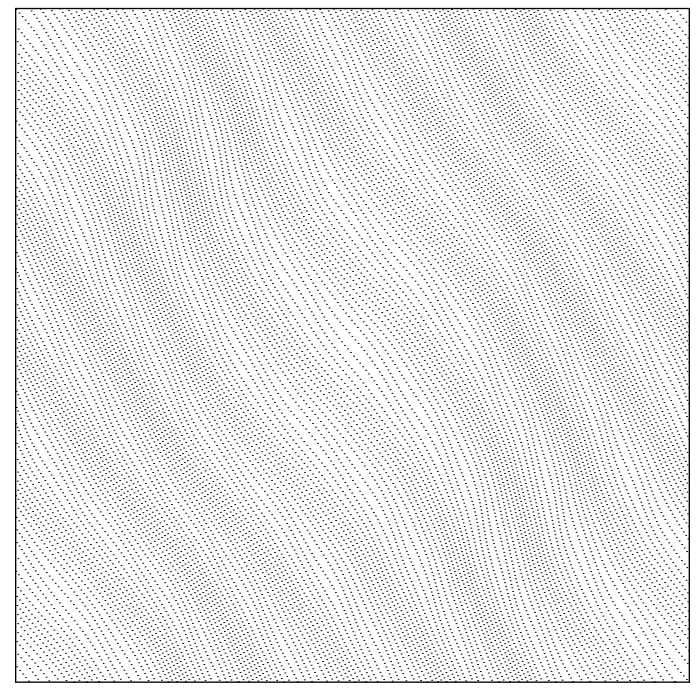

$$
k=0
$$

$\varepsilon=3.5$

$R=-5.41 \times 10^{-3}$

$$
\begin{array}{r}
k=0 \\
\varepsilon=3.6 \\
R=-2.05 \times 10^{3}
\end{array}
$$

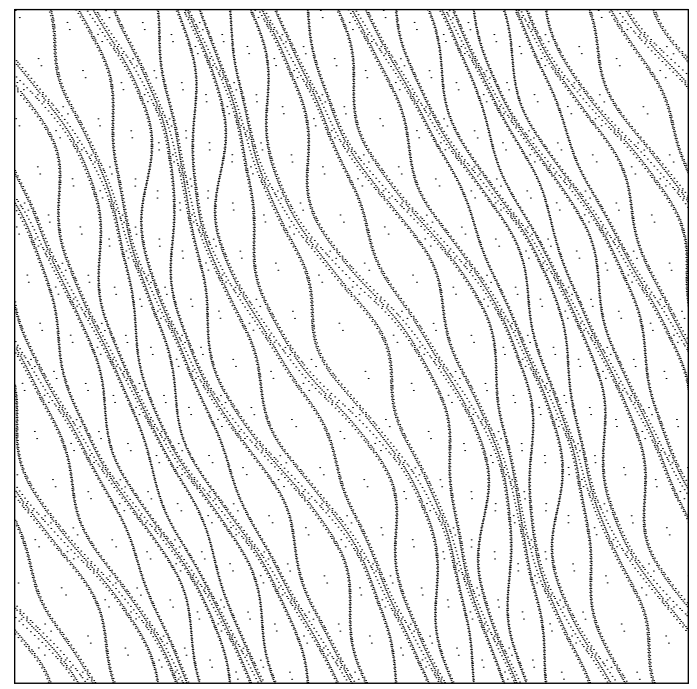

$$
\begin{aligned}
& k=0 \\
& \varepsilon=4 \\
& R=-1 \times 10^{78}
\end{aligned}
$$

FIGURE 5. Comparison of periodic orbits close to the invariant surface with rotation vector $(1,1)^{\infty}$, before and after breakdown. The periodic orbit rotation vector is $\left(\frac{10609}{19513}, \frac{16377}{19513}\right)$. On the top and middle rows, the orbit on the left, calculated using the indicated values of $k$ and $\varepsilon$, is approaching breakdown; for the one on the right, breakdown has already occurred. The orbit on the bottom row is a continuation along the path $k=0$ of the one on the middle row, right. Notice the abrupt increase in the value of the residue $R$. 
limit $N \rightarrow \infty$, to the Lyapunov exponent of the invariant set, as long as the orbit tends to the invariant set and motion on the invariant set is semiconjugate to rigid rotation with diophantine rotation vector (in which case there is only one invariant measure and it makes sense to talk of Lyapunov exponents without specifying the measure). Notice that, due to nature of the map, one eigenvalue of the derivative is always 1 and the other two are reciprocal.

Remark. It is important to monitor the algorithm that computes the periodic orbits to guarantee that the type of periodic orbit we are following is the same. As was remarked in [Olvera and Vargas 1992], periodic orbits of higher-dimensional symplectic maps may undergo bifurcations and follow a different (low-dimensional) torus from the one we are interested in.

To avoid this problem we have monitored the size of the condition numbers for the solution of the linear system (3.5). When the condition numbers were large we decreased the step size of the algorithm. Moreover we have tried to choose periodic orbits with rotation vectors whose components are irreducible, to avoid bifurcations similar to the ones observed in [Olvera and Vargas 1992]. The behavior of the stability exponent indicates that there were indeed bifurcations for some of the periodic orbits with reducible components, represented by the jumps in Figures 7 and 8 .

\section{CRITICAL FUNCTION}

Percival [1982] introduced a critical function for two-dimensional twist maps. It is a function of the rotation number and its value corresponds to the perturbation strength at which the invariant curve breaks down (in the case of the standard map there are indications from renormalizationgroup theory that breakdown occurs at a specific value for each rotation number). Based on the renormalization-group description, Buric, Percival, and Vivaldi [Buric et al. 1990] have observed that the critical function for the semistandard map has certain transformation properties under the modular group that allow its rapid computation. These transformation properties have been considered as additional evidence of an underlying renormalization-group transformation.

In analogy with [Percival 1982], we introduce a critical function $K(\omega)$ for the rotating standard map, from the space of rotation vectors $\omega$ to the parameter space $(\varepsilon, k)$. Given a rotation vector $\omega$, the values of the critical function are the values of the parameters at which breakdown occurs. As in the two-dimensional case, this function is discontinuous in a set of large measure (since it vanishes at all rationally dependent rotation vectors but is nonzero at a set of large measure).

We will use the value of the residue of a nearby periodic orbit as an indication of breakdown of an invariant surface.

In Figure 9 we present the logarithm of a slice of the critical function for the rotating standard map, at a fixed value of $k=0.2$. The figure was generated using periodic orbits and taking into account the symmetries of the critical function $\omega_{1} \mapsto$ $1-\omega_{1}, \omega_{2} \mapsto 1-\omega_{2}$. We chose the points at which to evaluate the critical function randomly, and then approximated the chosen points with a nearby (eventually) golden mean of the JacobiPerron algorithm (that is, with Jacobi-Perron expansion $\left.\left(\ldots,(1,1)^{\infty}\right)\right)$. We truncated the JacobiPerron expansion to obtain rational points close to the (eventual) golden means. All the periods used were between 300 and 800 . We used a continuation method to follow the periodic orbits up to the perturbation value of $\varepsilon$ at which the residue becomes 1. We made that perturbation strength the value of the critical function. (Cases when breakdown has already occurred for $\varepsilon=0$ are treated as if breakdown occurred at $\varepsilon=0$.) Our procedure is similar to the one used in [Buric et al. 1990] for the computation of the critical function for the semistandard map.

The computation of the data for Figure 9 required 30 hours of CPU time on an IBM RS/6000 370. The rendering was done using gnuplot, by 


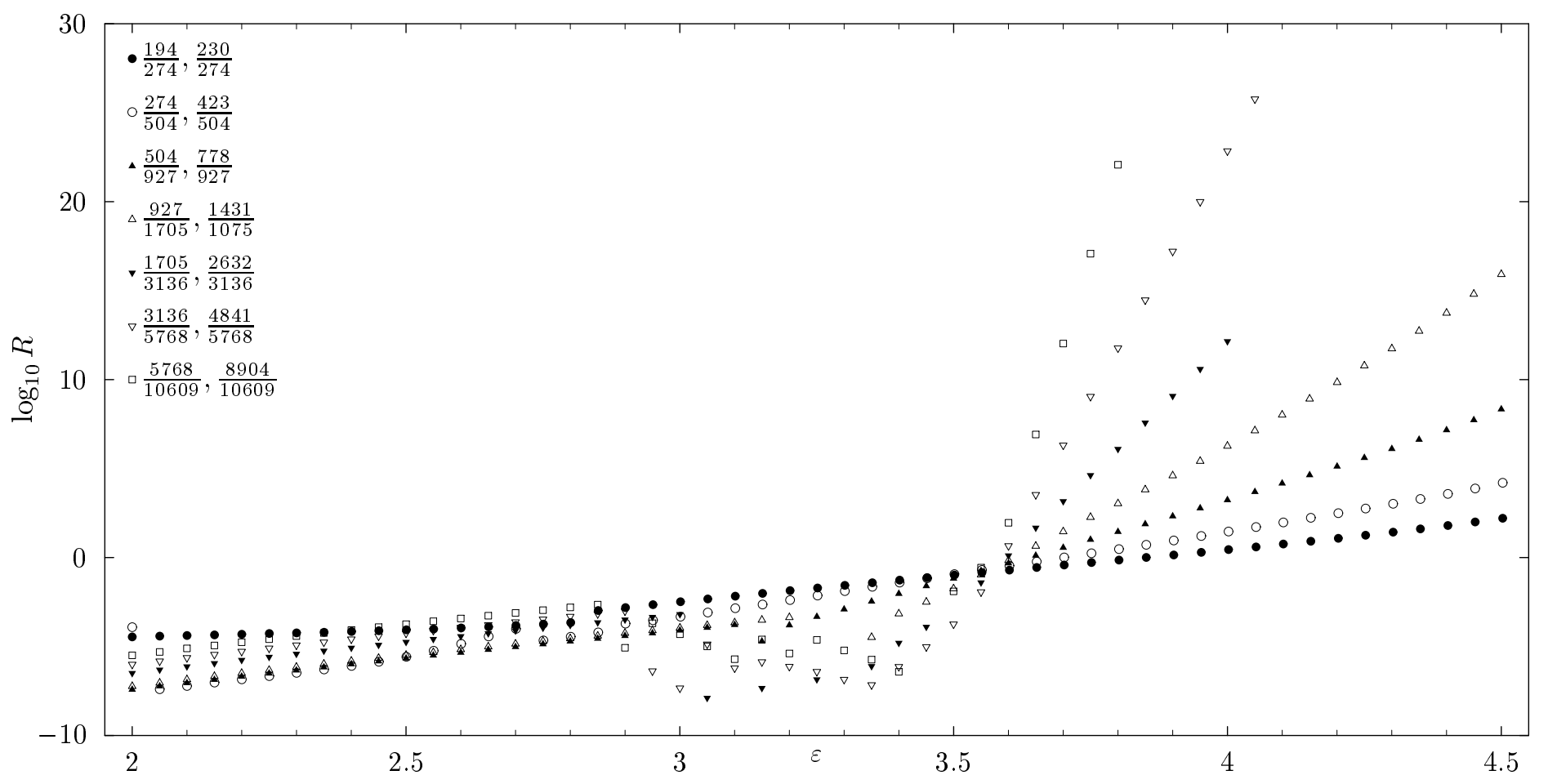

FIGURE 6. Residue of periodic orbits approximating an invariant set with rotation vector $(1,1)^{\infty}$ along the path $k=0$. The different sets correspond to different periodic orbits, with the ratios shown.

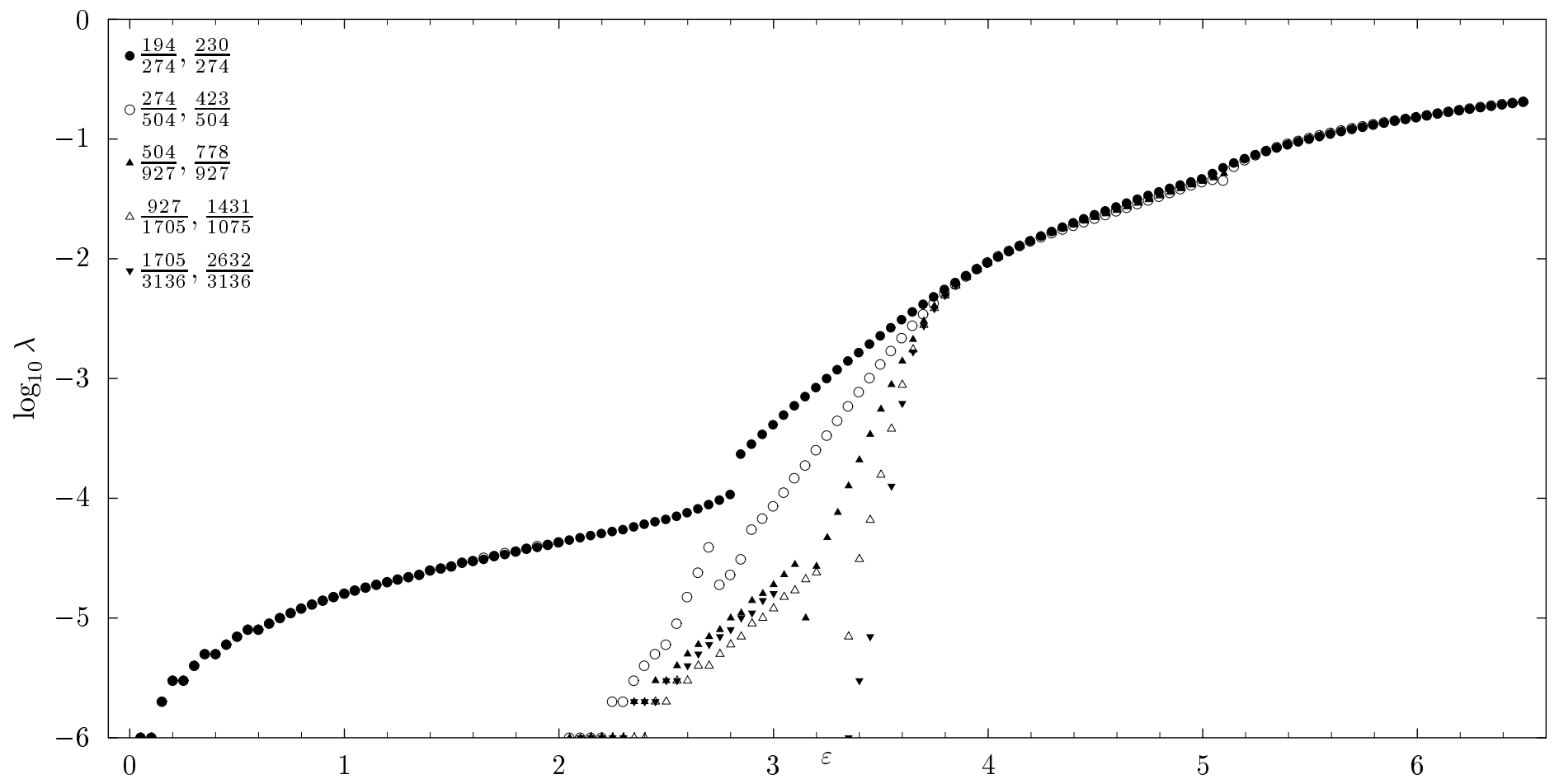

FIGURE 7. Stability index of the periodic orbits in the preceding figure. The jumps in the figure correspond to stability changes of the periodic orbit. 


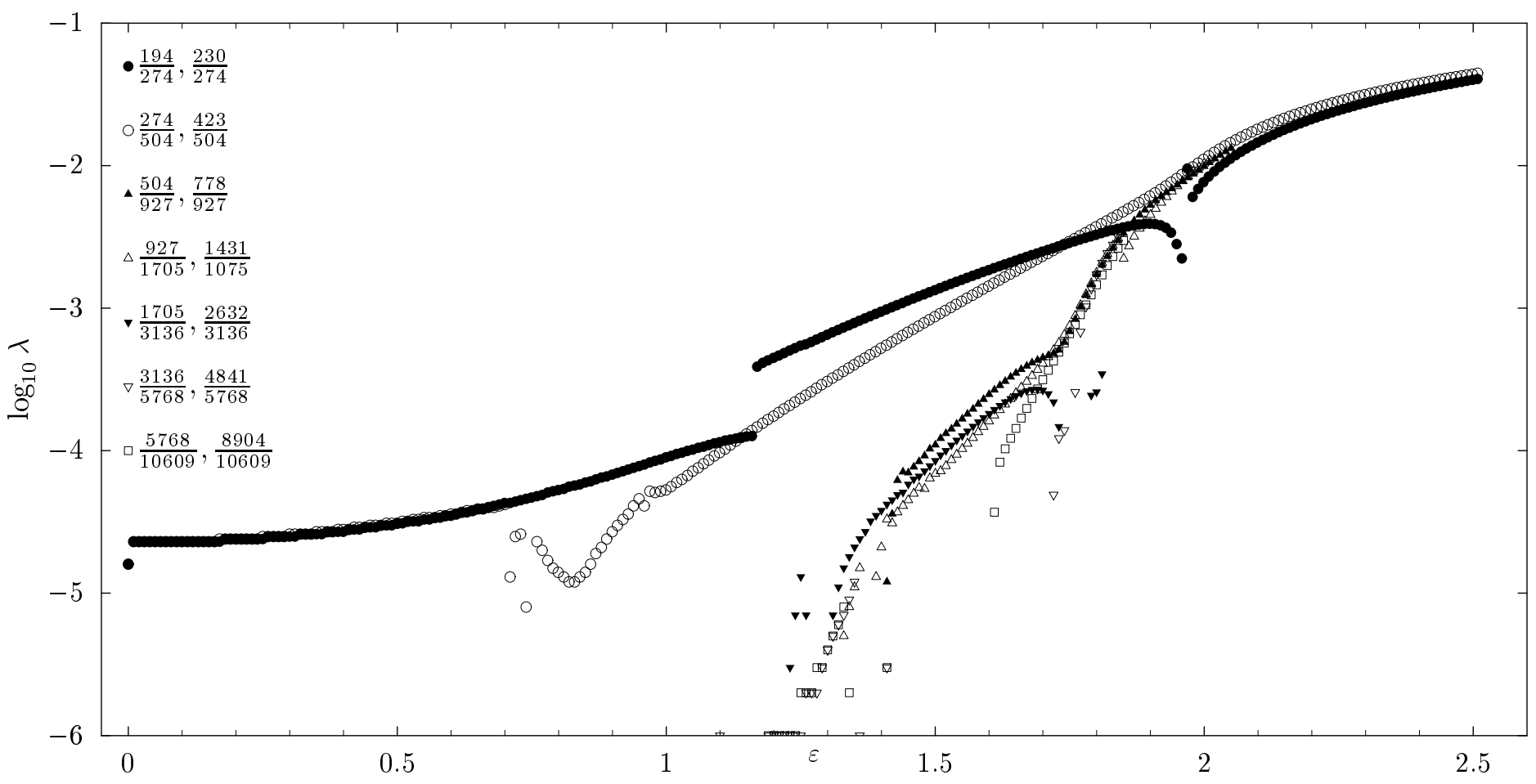

FIGURE 8. Stability index $\lambda$ of periodic orbits approximating an invariant set with rotation vector $(1,1)^{\infty}$ along the path $k=0.2$.

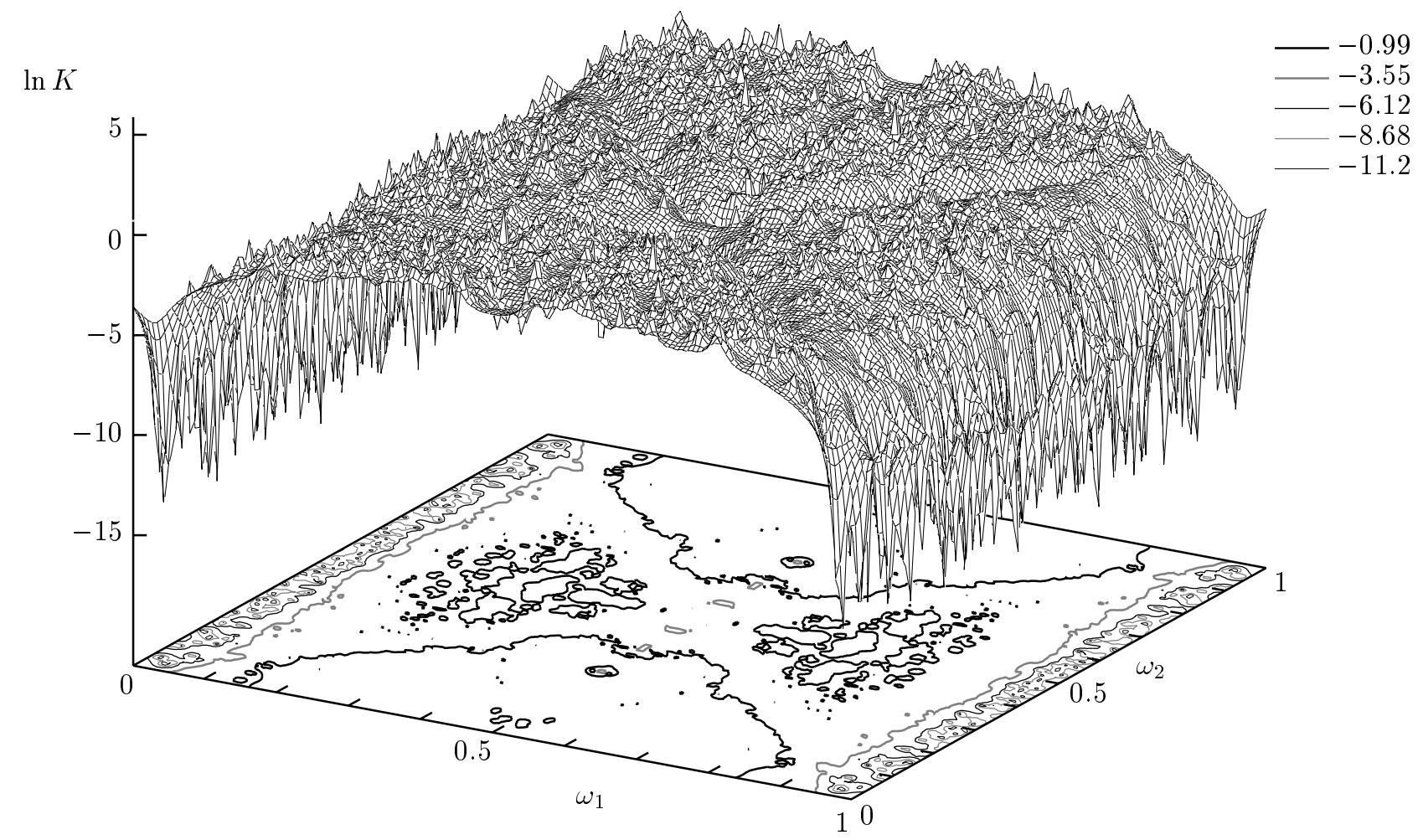

FIGURE 9. Logarithm of the critical function at $k=0.2$. The values of the function are computed using the residue of 6576 periodic orbits and interpolating on a $150 \times 150$ grid. Some level sets are also drawn. 
computing values at the grid points using weighted interpolation. (This process has the undesirable effect of "smoothing" the function in certain regions.)

We have not been able to identify transformation properties of the critical function under the action of the Jacobi-Perron operator for the rotating standard map. We face two problems, the first being a possible problem for any multi-dimensional approximation algorithm, while the second pertaining to the Jacobi-Perron algorithm in particular:

(a) We do not know how many parameters are necessary to describe the critical fixed point of a renormalization-group algorithm. In hopes of simple behavior (similar to the one uncovered by MacKay in the case of the twist maps of the annulus) we have used one-dimensional parameter paths in the parameter space. However, notice that, as indicated in Figure 1, there are regions in parameter space where the renormalization group behavior is more complicated.

(b) The Jacobi-Perron algorithm is not symmetric with respect to the two coordinates. In the case of twist maps (when the rotation vector is a real number) it is possible to produce a linear combination of $\log K(\omega)$ and $\log K(U \omega)$ to cancel the leading-order logarithmic singularities of the critical function. It would be interesting to determine whether such cancelations are possible in the case of the rotating standard map or high-dimensional symplectic maps.

\section{ACKNOWLEDGMENTS}

Part of this work was completed at Université de Rennes I.

I would like to thank Rafael de la Llave, Claudio Albanese, Luis Seco, John Im, Arturo Olvera, Jim Meiss and Robert MacKay for useful discussions and suggestions during this work. Finally I want to express my thanks to the people that developed and maintain gnuplot for making such a fine program available.

\section{REFERENCES}

[Artuso et al. 1991] R. Artuso, G. Casati, and D. L. Shepelyansky, "Breakdown of universality in renormalization dynamics for critical invariant torus", Europhys. Lett. 15 (1991), 381-386.

[Arnol'd 1963] V. I. Arnol'd, "Proof of a theorem of A. N. Kolmogorov on the invariance of quasi periodic motions under small perturbations of the Hamiltonian", Russ. Math. Surveys 18 (1963), 9.

[Arnol'd 1988] V. I. Arnol'd, Geometrical Methods in the Theory of Ordinary Differential Equations, 2nd ed., Springer, Berlin, 1988.

[Baldwin 1992] P. Baldwin, "A convergence exponent for multidimensional continued fraction algorithms", Jour. Stat. Phys. 66 (1992), 1507-1526.

[Bernstein 1971] L. Bernstein, The Jacobi-Perron Algorithm: Its Theory and Application, Lecture Notes in Math. 207, Springer, Berlin, 1971.

[Berretti and Chierchia 1990] A. Berretti and L. Chierchia, "On the complex analytic structure of the golden invariant curve for the standard map", Nonlinearity 3 (1990), 39-44.

[Buric et al. 1990] N. Buric, I. C. Percival, and F. Vivaldi, "Critical function and modular smoothing", Nonlinearity 3 (1990), 21-37.

[Celletti and Chierchia 1988] A. Celletti and L. Chierchia, "Construction of analytic K.A.M. surfaces and effective stability bounds", Comm. Math. Phys. 118 (1988), 119-161.

[Falcolini and Llave 1992a] C. Falcolini and R. de la Llave, "A rigorous partial justification of Greene's criterion", Jour. Stat. Phys. 67 (1992), 609-643.

[Falcolini and Llave 1992b] C. Falcolini, R. de la Llave, "Numerical calculation of domains of analyticity for perturbation theories in the presence of small divisors", Jour. Stat. Phys. 67 (1992), 645-666.

[Golé 1994] C. Golé, "Optical Hamiltonians and symplectic twist maps", Physica D71 (1994), 185195.

[Greene 1979] J. M. Greene, "A method for determining a stochastic transition", J. Math. Phys. 20 (1979), 1183-1201. 
[Kim and Ostlund 1986] S. Kim, S. Ostlund, "Simultaneous rational approximations in the study of dynamical systems", Phys. Rev. A34 (1986), 3426.

[Kincaid et al. 1982] P. R. Kincaid, J. R. Respess, D. M. Young, and R. G. Grimes, "ITPACK 2C: A FORTRAN package for solving large sparse linear systems by adaptive accelerated iterative methods", ACM Trans. on Math. Soft. 8 (1982), 302-322.

[Kolmogorov 1954] A. N. Kolmogorov, "On the conservation of conditionally periodic motions under small perturbations of the Hamiltonian", Dokl. Akad. Nauk. SSR 98 (1954), 469.

[Kook and Meiss 1989] H-T. Kook, J. D. Meiss, "Periodic orbits for reversible symplectic mappings", Physica D35 (1989), 65-86.

[Kosygin 1991] D. V. Kosygin, "Multidimensional KAM theory for the renormalization group viewpoint", pp. 99-129 in Dynamical Systems and Statistical Mechanics, edited by Ya. G. Sinai, Adv. Sov. Math. 3, Amer. Math. Soc., Providence, 1991.

[Lagarias 1993] J. C. Lagarias, "The quality of the diophantine approximations found by the JacobiPerron algorithm and related algorithms", Monatsh. Math. 115 (1993), 299-328.

[Llave 1993] R. de la Llave, "Introduction to K.A.M. theory", pp. 49-71 in Computational Physics (Almuñécar, 1992), edited by P. L. Garrido and J. Marro, World Scientific, Singapore and River Edge (NJ), 1993. Also available at http://rene.ma.utexas.edu/ mp_arc/papers.ext/93-8.tex.

[Llave and Rana 1991] R. de la Llave, D. Rana, "Accurate strategies for K.A.M. bounds and their implementation", pp. 127-146 in Computer Aided Proofs in Analysis, edited by K. Meyer and D. Schmidt, IMA Vol. Math. Appl. 28, Springer, New York, 1991.

[Mather 1991] J. N. Mather, "Variational construction of orbits of twist diffeomorphisms", Jour. Amer. Math. Soc. 4 (1991), 207-263.

[MacKay 1982] R. S. MacKay, "Renormalization in area preserving maps", Ph.D. thesis, Princeton University, 1982.

[MacKay 1992] R. S. MacKay, "On Greene's residue criterion", Nonlinearity 5 (1992), 161-187.
[Moser 1962] J. Moser, "On invariant curves of area preserving maps of an annulus", Nach. Akad. Wiss., Göttingen, Math. Phys. Kl. II 1 (1962), 1-20.

[Muldoon 1989] M. Muldoon, "Ghosts of order on the frontier of chaos", Ph.D. thesis, California Inst. of Technology, 1989.

[Olvera and Vargas 1992] A. Olvera and C. Vargas, "A continuation method to study periodic orbits of the Froeschlé map", preprint \#92-104, University of Texas at Austin, 1992. Available at http:// rene.ma.utexas.edu/mp_arc/papers.ext/92-104.ps.

[Percival 1982] I. C. Percival, "Chaotic boundary of a Hamiltonian map", Physica D6 (1982), 67-77.

[Poincaré 1892] H. Poincaré, Méthodes nouvelles de la mécanique celeste, 3 vols., Paris, Gauthier-Villars, 1892-99. English translation: New methods of celestial mechanics, edited by D. Goroff, History of modern physics and astronomy 13, Amer. Inst. Phys., Woodbury, NY, 1993.

[Perry and Wiggins 1994] A. D. Perry, S. Wiggins, "KAM tori are very sticky: rigorous lower bounds on the time to move away from an invariant Lagrangian torus with linear flow", Physica D71 (1994), 102-121.

[Press et al. 1992] W. H. Press, B. P. Flannery, S. Teukolski, and W. T. Vetterling, Numerical Recipes, 2nd ed., Cambridge Univ. Press, Cambridge, 1992.

[Rana 1987] D. Rana, "Proof of accurate upper and lower bounds to stability domains in small denominator problems", Ph.D. Thesis, Princeton University, 1987.

[Schweiger 1973] F. Schweiger, The metrical theory of Jacobi-Perron algorithm, Lect. Notes Math. 334, Springer, Berlin, 1973.

[Tompaidis 1996] S. Tompaidis, "Approximation of invariant surfaces by periodic orbits in high-dimensional maps: Some rigorous results", Experimental Mathematics 5 (1996), 197-209.

[Young and Gregory 1988] D. M. Young, R. T. Gregory, A survey of numerical mathematics, Dover, New York, 1988.

[Young and Hageman 1981] D. M. Young, L. A. Hageman, Applied Iterative Methods, Academic Press, New York, 1981. 
Stathis Tompaidis, Department of Mathematics, University of Toronto, 100 St. George Street, Toronto, Ontario M5S 1A1, Canada (stathis@math.toronto.edu)

Received November 14, 1995; accepted July 2, 1996 